\title{
Using Kolmogorov Complexity for Understanding Some Limitations on Steganography
}

\author{
Boris Ryabko*, Daniil Ryabko ${ }^{\dagger}$
}

October 31, 2018

\begin{abstract}
Recently perfectly secure steganographic systems have been described for a wide class of sources of covertexts. The speed of transmission of secret information for these stegosystems is proportional to the length of the covertext. In this work we show that there are sources of covertexts for which such stegosystems do not exist. The key observation is that if the set of possible covertexts has a maximal Kolmogorov complexity, then a high-speed perfect stegosystem has to have complexity of the same order.
\end{abstract}

\section{Introduction}

The goal of steganography can be described as follows. Alice and Bob can exchange messages of a certain kind (called covertexts) over a public channel. The covertexts can be, for example, a sequence of photographic images, videos, text emails and so on. Alice wants to pass some secret information to Bob so that Eve, the observer, cannot notice that any hidden information is being passed. Thus, Alice should use the covertexts to hide the secret text.

Cachin [1] suggested an information-theoretic model for steganography, along with a definition of a perfectly secure steganographic system. According to this model, Alice has an access to a probabilistic source of covertexts. It is usually assumed that the secret message can be represented as a sequence of independent equiprobable bits. She has to embed her secret message into covertexts in such a way that Bob can decode the message. A stegosystem is called perfectly secure if the distribution of the output is the same as the distribution at the source of covertexts. Indeed, in this case nobody is able to distinguish containers with hidden information and "empty" ones (i.e. without hidden information).

Let us consider an example of a perfect stegosystem suggested in [5]. The source of covertexts $\mu$ is as follows. It generates sequences of $n$ independently

*Institute of Computational Technologies of Siberian Branch of Russian Academy of Science Siberian State University of Telecommunications and Informatics, Novosibirsk, Russia boris@ryabko.net

${ }^{\dagger}$ INRIA Lille-Nord Europe daniil@ryabko.net 
identically distributed letters from some finite alphabet $A$, where $n \in \mathbb{N}$ is given. For the sake of simplicity we consider the binary alphabets $A=\{a, b\}$, but the construction can be used for the general case too [5]. The distribution $\mu$ can be unknown to Alice and Bob. Suppose that Alice has to transmit a secret sequence $y^{*}=y_{1} y_{2} \ldots$ generated by a source $\omega$, where $\omega\left(y_{i}=0\right)=1 / 2$ independently for all $i \in \mathbb{N}$, and let there be given a covertext sequence $x^{*}=x_{1} x_{2} \ldots$ generated by $\mu$. For example, let

$$
y^{*}=01100 \ldots, \quad x^{*}=a a b a b a a a a b b a a a a a b b
$$

The sequences $x^{*}$ and $y^{*}$ are encoded in a new sequence $X$ (to be transmitted to Bob) such that $y^{*}$ is uniquely determined by $X$, and the distribution of $X$ is the same as the distribution of $x^{*}$ (that is, $\mu$; in other words, $X$ and $x^{*}$ are statistically indistinguishable).

The encoding is carried out in two steps. First we group all symbols of $x^{*}$ into pairs, and denote

$$
a a=u, b b=u, a b=v_{0}, b a=v_{1} .
$$

In our example, the sequence (11) is represented as

$$
x^{*}=a a b a b a a a a b b a a a a a b b=u v_{1} v_{1} u v_{0} v_{1} u u u
$$

Then $X$ is acquired from $x^{*}$ as follows: all pairs corresponding to $u$ are left unchanged, while all pairs corresponding to $v_{k}$ are transformed to pairs corresponding to $v_{y_{1}} v_{y_{2}} v_{y_{3}}$; in our example

$$
X=a a a b b a a a b a a b a a a a b b
$$

Decoding is obvious: Bob groups the symbols of $X$ into pairs, ignores all occurrences of $a a$ and $b b$ and changes $a b$ to 0 and $b a$ to 1 .

The described stegosystem has the following properties. The sequence of symbols output by the stegosystem obeys the same distribution $\mu$ as the input sequence. The average length of the transmitted secret sequence is $n \mu(a b)$; in other words, the speed of transmission of hidden information is $\mu(a b)$ secret bits per letter of covertext. Moreover, in [5] a generalization of the described construction is proposed, for which the speed of transmission of secret text approaches the Shannon entropy $h(\mu)=-(\mu(a) \log \mu(a)+\mu(b) \log \mu(b))$ when $n$ goes to infinity. In addition, a similar construction is proposed in the same work for the case of arbitrary alphabets and for finite-alphabet Markov sources of covertexts.

So, we can see that perfectly secure stegosystems exist for a wide class of covertexts and, moreover, such systems are quite simple and have a high speed of transmission of secret information. Naturally, one is interested in the question of whether such stegosystems exist for any possible source of covertext. This problem is of interest since sources of covertexts that are of particular practical importance, such as texts in natural languages or photographs, do not seem to be well-described by any known simple model. Here we answer this 
question in the negative. More precisely, it turns out that there exists such a set of covertexts of length $n$ for which simple stegosystems which have speed of transmission of hidden text $\Omega(n)$ do not exist. Here simplicity is measured by Kolmogorov complexity of the system and a stegosystem is "simple" means that its complexity is $\exp (o(n))$, when $n$ goes to infinity. Kolmogorov complexity is an intuitive notion that often helps to establish results that help to understand the principled limitations a certain problem or model imposes; it has been used as such in many works, see, for example, [3, 6, 9, 10.

This result can be interpreted as that there are such complicated sources of data, that one cannot conceivably put significantly more information into a source, without changing its characteristics, even though the entropy of the source is very high. This may explain what is known in practice; for example, it is apparently very hard to put any hidden message into a given text in a natural language, without making the text "unnatural". Of course, rather than trying to change a given text, the communicating parties can easily agree in advance on two texts each of which codes one secret bit, so that when the need for communication arises, Alice can transmit one of the texts thereby passing one bit. However, in order to communicate more than one bit, to use the same method they would have to have a database of covertexts that is exponentially large with respect to the message to pass. Moreover, even this stegosystem will not be perfectly secure, since the source of covertexts with hidden information is concentrated on a small subset of all the possible covertexts of the given length. If the stegosystem is used once, then perhaps no reliable detection of the hidden message is possible. If it is to be used on multiple occasions, that is, if we wish to construct a general purpose stegosystem for transmitting, say, $\delta n$ bits with an $n$-bit message (for some fixed $\delta$ ), we will need to construct a database of effectively all possible covertexts. At least, this is the case for some sources of covertexts, as the result of this work demonstrates, and it seems likely that it is the case for such sources as texts in natural languages or even photographic images. Thus, our result may be helpfull in clarifying the nature of the difficulties that arise in construction of real steganographic systems which use human-generated sources of covertexts.

\section{Preliminaries}

We use the following model for steganography, mainly following [1. It is assumed that Alice has an access to an oracle which generates covertexts according to some fixed but unknown distribution of covertexts $\mu$. Covertexts belong to some finite alphabet $A$. For the sake of simplicity we consider the case $A=\{0,1\}$; the general case is analogous. Alice wants to use this source to transmit hidden messages. A hidden (or secret) message is a sequence of letters from $\{0,1\}$ generated independently with equal probabilities of 0 and 1 . We denote the source of hidden messages by $\omega$. This is a commonly used model for the source of secret messages, since it is assumed that secret messages are encrypted by Alice using a key shared only with Bob. If Alice uses the Vernam cipher (a 
one-time pad) then the encrypted messages are indeed generated according to the Bernoulli 1/2 distribution, whereas if Alice uses modern block or stream ciphers then the encrypted sequence "looks like" a sequence of random Bernoulli $1 / 2$ trials. (Here "looks like" means indistinguishable in polynomial time, or that the likeness is confirmed experimentally by statistical data, see, e.g. 44.) The third party, Eve is a passive adversary: Eve is reading all messages passed from Alice to Bob and is trying to determine whether secret messages are being passed in the covertexts or not. Clearly, if covertexts with and without hidden information have the same probability distribution $(\mu)$ then it is impossible to distinguish them.

Since the number of possible covertexts $x$ in the set $A^{n}$ is finite, using a stegosystem once Alice can only transmit a finite number of bits of the secret message. We tacitly assume that there are always more secret bits than Alice wants to pass, which is formalized by assuming that Alice has an infinite secret message (in practice, if Alice runs out of secrets, she can fill the remainder of the message with random noise). Depending on the covertext that Alice has and on the actual secret message, the length of the secret text that she transmits may vary. Naturally, one wishes to maximize the expected length of the secret message that the encoder can transmit. We require, however, that the decoding is always correct, that is, Bob gets the whole secret message that Alice has transmitted, without errors.

The steganographic protocol can be summarized in the following definitions.

Definition 1 (secret or hidden text). A source $\omega$ of secret text $y^{*}=y_{1}, y_{2}, \ldots$ is such that $\omega\left(y_{i}=0\right)=\omega\left(y_{i}=1\right)=1 / 2$, independently for all $i \in \mathbb{N}$.

Definition 2 (stegosystem). A stegosystem $\mathrm{St}$ is a family (indexed by $n$ ) of pairs of functions: the encoder, that maps a pair $\left(x, y^{*}\right) \in A^{n} \times\{0,1\}^{\infty}$ (a covertext and a secret sequence) into a pair $\left(t, \operatorname{StEnc}_{n}\left(x,\left(y_{1} \ldots y_{t}\right)\right) \in \mathbb{N} \times A^{n}\right.$ : the number of secret bits transmitted and the output covertext. The decoder $\mathrm{StDec}_{n}$ is a function from $A^{n}$ to $\{0,1\}^{*}$. We will often omit the parameter $n$ from the notation, when its value is clear.

Definition 3 (steganographic protocol). A parameter $n$ is fixed. Alice draws a covertext $x \in A^{n}$ generated by a source of covertexts $\mu$ (a distribution on $A^{n}$ ) and a secret message $y^{*}=y_{1}, y_{2}, \ldots$ according to the source $\omega$. The sources $\omega$ and $\mu$ are independent of each other.

Given $x \in A^{n}$ and $y^{*}$ Alice using a stegosystem St obtains the number of secret bits she can pass $t\left(x, y^{*}\right) \geq 0$, and a covertext $\operatorname{StEnc}\left(x,\left(y_{1} \ldots y_{t}\right)\right) \in A^{n}$ that is transmitted over a public channel to Bob. (Only $\operatorname{StEnc}\left(x,\left(y_{1} \ldots y_{t}\right)\right)$ is transmitted; the number $t$ is not.)

Bob (and any possible observer Eve) receives $x^{\prime} \in A^{n}$ and obtains using the decoder StDec the resulting message $\operatorname{StDec}_{n}\left(x^{\prime}\right)=y_{1} \ldots y_{t}$.

Definition 4 (perfect security). A steganogrpahic system is called perfectly secure if the sequence of covertexts $x^{*}$ and the steganographic sequence $X$ have the same distribution: $\operatorname{Pr}_{\mu \times \omega}\left(\mathrm{StEnc}=x^{\prime}\right)=\mu\left(x^{\prime}\right)$ for any $x^{\prime} \in A^{n}$, where the 
first probability is taken with respect to the distribution of covertexts $\mu$ and that of secret text $\omega$.

Definition 5 (speed of transmission). For a stegosystem St the speed of transmission of secret text $v_{n}(\mathrm{St})$ is defined as $\mathbf{E}_{\mu \times \omega} t\left(x, y^{*}\right) / n$ (the expectation is with respect to $\mu$ and $\omega)$.

Note that often (in particular, in [1) more general steganographic protocols are considered, allowing for non-perfect security, transmission with errors, several draws from the source of covertexts, etc. We have decided to concentrate on the simple model presented since it is rich enough for perfectly secure stegosystems to exist, for a wide classes of sources of covertexts (e.g. all finite-memory sources, [5]). Some possible extensions are discussed in Section 4

For definitions, notation, and an introduction to Kolmogorov complexity, see [3. Informally, the Kolmogorov complexity, or algorithmic entropy, can be defined as follows [8]: $K(x)$ of a string $x$ is the length (number of bits) of a shortest binary program (string) to compute $x$ on a fixed reference universal computer (such as a particular universal Turing machine). Intuitively, $K(x)$ represents the minimal amount of information required to generate $x$ by any effective process. The conditional Kolmogorov complexity $K(x \mid y)$ of $x$ relative to $y$ is defined similarly as the length of a shortest program to compute $x$, if $y$ is furnished as an auxiliary input to the computation.

We will use some simple properties of $K$, such as $K(s) \leq|s|+c$ for any word $s$, whose proofs can be found in e.g. 3. Here it is worth noting that $K(s)$ does not take into account the time or extra memory it takes to compute $s$.

\section{Main results}

Theorem 1. For every $\delta>0$ there is a family indexed by $n \in \mathbb{N}$ of distributions $P_{n}$ on $A^{n}$ with $h\left(P_{n}\right) \geq n-1$, such that every stegosystem $\mathrm{St}_{n}$ whose Kolmogorov complexity satisfies $\log K\left(S t_{n}\right)=o(n)$ and whose speed of transmission of hidden text $v_{n}\left(\mathrm{St}_{n}\right)$ is not less than $\delta$, is not perfectly secure from some $n$ on.

Proof. We will construct a sequence of sets $X_{n}$ of words of length $n$ whose Kolmogorov complexity is the highest possible, namely $2^{\Omega(n)}$. For each $n \in \mathbb{N}$, the distribution $P_{n}$ is uniform on $X_{n}$. We will then show that, in order to have the speed of transmission $\delta>0$ a perfectly secure stegosystem must be able to generate a large portion of the set $X_{n}$, for each $n$. This will imply that the complexity of such a stegosystem has to be $2^{\Omega(n)}$. The latter implication will be shown to follow from the fact that, in order to transmit some information, a stegosystem must replace the input with some output that could have been generated by the source; this, for perfectly secure stegosystems, amounts to knowing at least a large portion of $X_{n}$.

Fix $n \in \mathbb{N}$ and let $X \subset A^{n}$ be any set such that $|X|=2^{n-1}$ and

$$
K(X)=2^{n}(1+o(1))
$$


The existence of such a set can be shown by a direct calculation of the number of all subsets with $2^{n-1}$ elements; the maximal complexity is equal (up to a constant) to the log of this value.

Assume that there is a perfectly secure stegosystem $S t_{n}$ for the family $P_{n}$, $n \in \mathbb{N}$, and let the speed of transmission of hidden text be not less than $\delta$. Define the set $Z$ as the set of those words which are used as codewords $Z:=$ $\left\{x \in A^{n}: \operatorname{StDec}(x) \neq \Lambda\right\}$. Since the expected speed of transmission of hidden text is lower bounded by $\delta$, we must have $|Z| \geq \delta 2^{n-1}$ (indeed, since every word codes at most $n-1$ bits, the expected speed of transmission must satisfy $\left.(n-1) \frac{|Z|}{2^{n-1}} \geq \delta n\right)$. Since St is perfectly secure $Z \subset X$. Let us lower-bound the complexity $K(Z \mid X \backslash Z)$ of the set $Z$ given $X \backslash Z$. Given the description of $X \backslash Z$ and the description of $Z$ relative to $X \backslash Z$ one can reconstruct $X$. That is why $K(Z \mid X \backslash Z) \geq K(X)-K(X \backslash Z)+O(1)$. The size of $X \backslash Z$ is not greater than $2^{n-1}(1-\delta)$. Hence,

$$
K(Z \mid X \backslash Z) \geq K(X)-\max _{|U| \leq 2^{n-1}(1-\delta)} K(U)+O(1)
$$

The latter maximal complexity can be calculated as follows:

$$
\max _{|U| \leq 2^{n-1}(1-\delta)} K(U)=\log \left(\begin{array}{c}
2^{n} \\
2^{n-1}(1-\delta)
\end{array}\right)+O(1) .
$$

Applying the Stirling approximation for factorial we obtain

$$
\max _{|U| \leq 2^{n-1}(1-\delta)} K(U) \leq 2^{n}(1-\gamma)(1+o(1))
$$

where $\gamma=1-h\left(\frac{1-\delta}{2}, \frac{1+\delta}{2}\right)$. From this equality, (2) and (3) we get

$$
K(Z) \geq \gamma 2^{n}(1+o(1)) .
$$

Furthermore, define $Z_{0}$ as the set of words that code those secret messages that start with 0 , and $Z_{1}$ those that start with 1 :

$$
Z_{i}:=\left\{x \in A^{n}: \operatorname{StDec}(x)=i u, u \in\{0,1\}^{*}\right\}, i \in\{0,1\} .
$$

Clearly, $Z=Z_{1} \cup Z_{0}$. Hence, $K(Z \mid X \backslash Z) \leq K\left(Z_{0} \mid X \backslash Z\right)+K\left(Z_{1} \mid X \backslash Z\right)+O(1)$, so that $K\left(Z_{i} \mid X \backslash Z\right) \geq K(Z \mid X \backslash Z) / 2+O(1)$ for some $i \in\{0,1\}$. Let this $i$ be 1 . Thus,

$$
K\left(Z_{1} \mid X \backslash Z\right) \geq \gamma 2^{n-1}(1+o(1)) .
$$

We will next show how to obtain $Z_{1}$ from $Z \backslash Z_{1}$ and the stegosystem St, thus arriving at a contradiction with the assumption that $\log K(\mathrm{St})=o(n)$.

For a set $T \subset X$ define

$$
\varphi(T):=\left\{\operatorname{StEnc}(x, 1 u): x \in T, u \in\{0,1\}^{*}\right\} .
$$

Since St is perfectly secure, $\varphi(T) \subset X$ for every $T \subset X$. Let $T_{0}=X \backslash Z_{1}$, and $T_{k}=T_{k-1} \cup \varphi\left(T_{k-1}\right)$. Since $X$ is finite and each $T_{k-1}$ is a subset of $T_{k}$, there 
must be such $k_{0} \in \mathbb{N}$ that $T_{k}=T_{k_{0}}$ for all $k>k_{0}$. There are two possibilities: either $T_{k_{0}}=X$ or $X \backslash T_{k_{0}} \neq \varnothing$. Assume the latter, and define $Z_{1}^{\prime}=X \backslash T_{k_{0}}$. Then to obtain an element of $Z_{1}^{\prime}$ as an output of the stegosystem St, the input must be an element of $Z_{1}^{\prime}$ and a secret message that starts with 1. From this, and from the fact that the distribution of the output is the same as the distribution of the input (that is, St is perfectly secure), we get

$$
P_{n}\left(Z_{1}^{\prime}\right)=P_{n}\left(Z_{1}^{\prime}, y=1 u\right)=P_{n}\left(Z_{1}^{\prime}\right) \omega(1)=P_{n}\left(Z_{1}^{\prime}\right) / 2,
$$

which implies $P_{n}\left(Z_{1}^{\prime}\right)=0$ and $Z_{1}^{\prime}=\varnothing$. Therefore, there is a $k \in \mathbb{N}$ such that $T_{k}=X$. This means that a description of $Z_{1}$ can be obtained from a description of $X \backslash Z_{1}=T_{0}$ and St. Indeed, to obtain $Z_{1}$ it is sufficient to run StEnc on all elements of $T_{0}$ with all inputs starting with 1 , thus obtaining $T_{1}$, and then repeat this procedure until we get $T_{k+1}=T_{k}$ for some $k$, wherefrom we know that $T_{k}=X$ and $Z_{1}=T_{k} \backslash T_{0}$. Thus,

$$
K\left(Z_{1} \mid X \backslash Z_{1}\right) \leq K(\mathrm{St})+O(1)=2^{o(n)}
$$

which contradicts (5).

\section{Possible extensions}

The definitions of stegosystems and steganographic protocol that we have used allow for several extensions. In particular, we have made the requirement that Alice can draw only one covertext from the source, in order to construct her message. We have also required that the decoding is always correct, did not allow for a secret key in the protocol (a secret key could be used before entering into steganographic communication in order to obtain the secret message $y^{*}$, but is out of scope of the protocol), etc. These requirements, along with the requirement of perfect security, might be considered restrictive; however, as was mentioned in the Introduction, for some sources of covertexts (such as i.i.d. or finite-memory sources) there are indeed perfectly secure steganographic systems that meet all the requirements we have made, and which also have the highest possible speed of transmission of hidden text: $v_{n}(\mathrm{St})$ approaches (exponentially fast) the Shannon entropy $h(\mu)$ of the source of covertexts, as $n$ grows (see [5]). This is why we have decided to sacrifice the generality for the sake of simplicity of the model presented.

Nevertheless, it is worth noting that the main results of this work can be extended to more general cases. For example, if we allow Alice and Bob to share a secret key $k_{n}$, then trivially Theorem 1 holds with $K\left(\mathrm{St}_{n}\right)$ replaced by $K\left(\mathrm{St}_{n}\right)+K\left(k_{n}\right)$. Let us briefly stop on another extension of the protocol. Instead of allowing Alice to draw only one covertext from the source, we can allow her to draw several, say, $M$ covertexts. Given $M$ covertexts $x_{1}, \ldots, x_{m}$, where $x_{i} \in A^{n}$, and a secret sequence $y^{*}$ Alice constructs a single $x^{\prime} \in A^{n}$ which is passed (over a public channel) to Bob. In particular, depending on the message $y^{*}$, Alice can chose $x_{i}$ that already encodes the message, if such $x_{i}$, 
$1 \leq i \leq M$ exists. The speed $v_{n}$ of transmission of secret text is measured with respect to what is passed over the public channel only (i.e. $x^{\prime}$ ). Then Theorem 1 admits the following extension: there are such sources of covertexts, that any perfectly secure simple stegosystem must draw $M_{n}=2^{\Omega(n)}$ covertexts in order to transmit $\delta n$ bits, for any given $\delta>0$.

\section{References}

[1] Cachin C. An information-theoretic model for steganography. Information and Computation, v. 192, pp. 41-56, 2004. Also in: Proc. 2nd Information Hiding Workshop, v. 1525 of LNCS, pp. 306-318, Springer Verlag, 1998.

[2] Kolmogorov, A.N. Three approaches to the quantitative definition of information, Problems of Inform. Transmission, 1, 1965, 3-11.

[3] Li, M., Vitanyi, P.: An Introduction to Kolmogorov Complexity and Its Applications, Springer-Verlag, New York, 2nd Edition, 1997.

[4] Ryabko B., Fionov A. Basics of Contemporary Cryptography for IT Practitioners. World Scientific Publishing Co., 2005.

[5] Ryabko, B. and Ryabko, D. Information-theoretic approach to steganographic systems. In Proc. 2007 IEEE International Symposium on Information Theory (ISIT'07), pages 2461-2464, Nice, France, 2007. IEEE. See an extended version in http://arxiv.org/abs/0809.1593.

[6] Uspenskii V.A.,Semenov A.L.,Shen A.K. Can an individual sequence of zeros and ones be random?, Russian Mathematical Surveys, 45, 1990.

[7] Vereshchagin N., Vitanyi P.M.B., Kolmogorov's structure functions with application to the foundations of model selections. In: Proc. 43th Symposium on Foundations of Computer Science, 2002, 751- 760.

[8] Vitanyi P.M.B., Meaningful Information, IEEE Trans. Inform. Theory, 52( 10), 2006, 4617-4626.

[9] Vitanyi, P.M.B., Li, M. Minimum description length induction, Bayesianism, and Kolmogorov complexity, IEEE Trans. Inform. Theory, 46(2), 2000, 446464 .

[10] V'yugin V.V. Suboptimal measures of predictive complexity for absolute loss function. Information and Computation. 2002, v. 175, p. 146-157. 